\title{
A New Model for Time-delay Systems with Application to Network Based Control
}

\author{
Huijun $\mathrm{Gao}^{1,2}$, Tongwen Chen ${ }^{2}$, James Lam ${ }^{3}$ \\ 1. Space Control and Inertial Technology Research Center, Harbin Institute of Technology, Harbin 150001, China \\ E-mail: hjgao@hit.edu.cn \\ 2. Department of Electrical and Computer Engineering, University of Alberta, Edmonton, Alberta, Canada T6G 2V4 \\ E-mail: hjgao, tchen@ece.ualberta.ca \\ 3. Department of Mechanical Engineering, University of Hong Kong, Hong Kong \\ E-mail: james.lam@hku.hk
}

\begin{abstract}
This paper proposes a new model for time-delay systems, which contains multiple successive delay components in the state and has important applications in remote control and network based control. New results on stability and $\mathscr{H}_{\infty}$ performance are obtained for systems with two successive delay components. The second part of this paper applies the proposed new model to network based control, which has emerged as a topic of significant interest in the control community. A sampled-data networked control system with simultaneous consideration of network induced delays, data packet dropouts and measurement quantization is modelled as a time-delay system with two successive delay components in the state and, the problem of network based $\mathscr{H}_{\infty}$ control is solved accordingly.
\end{abstract}

Key Words: Networked control, sampled-data systems, time-delay systems

\section{Motivation and New Model}

Time-delay systems, also called systems with aftereffect, equations with deviating argument or differentialdifference equations, have kept being an active research area for the last few decades. The main reason is that many processes include after-effect phenomena in their inner dynamics, and engineers require models to behave more like real processes due to the ever-increasing expectations of dynamic performance. There is a great number of research results concerning time-delay systems scattered in the literature (see, $[4,6,7,8,9,10,11,12,18]$ and the references therein).

The most commonly and frequently used state-space model to represent time-delay systems is

$$
\dot{x}(t)=A x(t)+A_{d} x(t-d(t)),
$$

where $d(t)$ is a time delay in the state $x(t)$, which is often assumed to be either constant, or time-varying satisfying certain conditions, e.g.,

$$
0 \leq d(t) \leq \bar{d}<\infty, \quad \dot{d}(t) \leq \tau<\infty
$$

Almost all the reported results on time-delay systems are based on this basic mathematical model. It is noted that in

IEEE Catalog Number: 06EX1310

This work was supported by an Alberta Ingenuity Fellowship, a Killam Memorial Postdoctoral Fellowship, and NSERC, Canada. This work was also partially supported by the Program for New Century Excellent Talents in University, and RGC Grant HKU 7028/04P. this model, the time delay in the state variable $x(t)$ is assumed to appear in a single and simple form. Sometimes in practical situations, however, signals transmitted from one point to another may experience a few segments of networks, which can possibly induce successive delays with different properties due to variable network transmission conditions. Let us consider, for example, a state-feedback remote control problem. Suppose the physical plant is given by $\dot{x}(t)=A x(t)+B u(t)$, and suppose the measurement of $x(t)$ travels through $s$ segments of transmission media, each of which will cause some delay with different properties, denoted as $d_{i}(t), i=1, \ldots, s$. In this case, the closed-loop system with state-feedback gain $K$ is given by

$$
\dot{x}(t)=A x(t)+B K x\left(t-\sum_{i=1}^{s} d_{i}(t)\right),
$$

and a natural assumption on the time delay $d_{i}(t)$ can be given by

$$
0 \leq d_{i}(t) \leq \bar{d}_{i}<\infty, \quad \dot{d}_{i}(t) \leq \tau_{i}<\infty .
$$

It can be seen that there are $s$ successive delay components appearing in the state $x(t)$. Since the properties of these delay components may not be identical due to the transmission conditions, it is not reasonable to combine them together. System (3) is what we refer to as systems with successive delay components.

One may argue that (3) with assumption (4) can be easily transformed into the form of (1) with assumption (2) by imposing

$$
\bar{d}=\sum_{i=1}^{s} \bar{d}_{i}, \quad \tau=\max _{i}\left(\tau_{i}\right) .
$$


However, it is not difficult to understand that such treatment will be conservative, especially when the delays $d_{i}(t)$, $i=1, \ldots, s$, have quite different properties. For illustration, let us assume that $\tau_{1}<\tau_{2}<\cdots<\tau_{s}$, then from (5) we have $\tau=\tau_{s}$. If we use the single delay model in (1), the delay parameters that can be used are $\bar{d}$ and $\tau$. In such a way, we have lost the parameters $\tau_{i}, i=1, \ldots, s-1$. More specifically, if we use some established stability results on delay systems (such as the linear matrix inequality (LMI) based one given in [14]), the delay-dependent LMI stability condition only contains the parameters $\bar{d}$ (or $\sum_{i=1}^{s} \bar{d}_{i}$ ) and $\tau$ (or $\tau_{s}$ ), and thus the parameters $\tau_{i}, i=1, \ldots, s-1$, will not be taken into consideration. This is more obvious for the case when some of $d_{i}(t)$ are constant and some are time-varying. Based on the above discussion, in this paper, our purpose is to introduce the new model in (3), and present new results for systems with successive delay components. To make our idea more lucid and to avoid complicated notation, we consider the case in which only two successive delay components appear in the state, and the idea behind this paper can be easily extended to systems with multiple successive delay components. An illustrative example is provided to show the significant advantage of this new model and the proposed stability condition over some existing single delay approaches. These constitute the contents of Section 2.

In Section 3, we apply the proposed new model to the problem of network based control, which has emerged as a topic of significant interest in the control community (see, $[1,13,22]$ and the references therein). As can be seen later, a sampled-data networked control system with simultaneous consideration of network induced delays, data packet dropouts and measurement quantization can be modelled as a time-delay system with two successive delay components in the state, which forms a solid background for the new model presented in this paper. Then, the $\mathscr{H}_{\infty}$ performance condition developed in Section 2 is exploited to investigate the problem of network based $\mathscr{H}_{\infty}$ control. An Illustrative example is provided to show the advantage and applicability of the developed results for network based controller design.

For space consideration, all proofs have been omitted, but can be found in the full version of the paper.

\section{Main Results}

\subsection{Stability Analysis}

Consider the following system with two successive delay components in the state:

$$
\begin{aligned}
\Sigma: \quad \dot{x}(t) & =A x(t)+A_{d} x\left(t-d_{1}(t)-d_{2}(t)\right), \\
x(t) & =\phi(t), \quad t \in[-\bar{d}, 0] .
\end{aligned}
$$

Here $x(t) \in \mathbb{R}^{n}$ is the state vector; $d_{1}(t)$ and $d_{2}(t)$ represent the two delay components in the state; $A, A_{d}$ are system matrices with appropriate dimensions. It is assumed that $d_{1}(t)$ and $d_{2}(t)$ satisfy (4), and $\bar{d}=\bar{d}_{1}+\bar{d}_{2} . \phi(t)$ is the initial condition on the segment $[-\bar{d}, 0]$.
In the following, we present a new stability criterion, which can be proved based on the following Lyapunov-Krasovskii functional:

$$
\begin{aligned}
V(t)= & V_{1}(t)+V_{2}(t)+V_{3}(t)+V_{4}(t), \\
V_{1}(t)= & x^{T}(t) P x(t), \\
V_{2}(t)= & \int_{t-d_{1}(t)}^{t} x^{T}(s) Q_{1} x(s) d s \\
& +\int_{t-d_{1}(t)-d_{2}(t)}^{t-d_{1}(t)} x^{T}(s) Q_{2} x(s) d s, \\
V_{3}(t)= & \int_{t-\bar{d}_{1}-\bar{d}_{2}}^{t} x^{T}(s) R x(s) d s, \\
V_{4}(t)= & \int_{-\bar{d}_{1}}^{0} \int_{\beta}^{0} \dot{x}^{T}(t+\alpha) Z_{1} \dot{x}(t+\alpha) d \alpha d \beta \\
& +\int_{-\bar{d}_{1}-\bar{d}_{2}}^{-\bar{d}_{1}} \int_{\beta}^{0} \dot{x}^{T}(t+\alpha) Z_{2} \dot{x}(t+\alpha) d \alpha d \beta \\
& +\int_{-\bar{d}_{1}-\bar{d}_{2}}^{0} \int_{\beta}^{0} \dot{x}^{T}(t+\alpha) M \dot{x}(t+\alpha) d \alpha d \beta,(7)
\end{aligned}
$$

where $P>0, Q_{1} \geq Q_{2} \geq 0, R \geq 0, Z_{1} \geq Z_{2}>0$ and $M>0$ are matrices to be determined.

Theorem 1 System $\Sigma$ in (6) with delays $d_{1}(t)$ and $d_{2}(t)$ satisfying (4) is asymptotically stable if there exist matrices $P>0, Q_{1} \geq Q_{2} \geq 0, R \geq 0, Z_{1} \geq Z_{2}>0, M>0$, and $S, T$, $U, V$ satisfying

$$
\left[\begin{array}{cc}
\Xi_{1}+\Xi_{2}+\Xi_{2}^{T}+\Xi_{3} & \Xi_{4} \\
* & \Xi_{5}
\end{array}\right]<0,
$$

where

$$
\begin{aligned}
& \Xi_{1}=\left[\begin{array}{cccc}
\Delta_{1} & 0 & P A_{d} & 0 \\
* & \Delta_{2} & 0 & 0 \\
* & * & \Delta_{3} & 0 \\
* & * & * & -R
\end{array}\right], \\
& \Xi_{2}=\left[\begin{array}{llll}
S+V & T-S & U-T & -U-V
\end{array}\right], \\
& \Xi_{3}=\Delta_{4}^{T}\left[\bar{d}_{1} Z_{1}+\bar{d}_{2} Z_{2}+\left(\bar{d}_{1}+\bar{d}_{2}\right) M\right] \Delta_{4}, \\
& \Xi_{4}=\left[\begin{array}{llll}
S & T & U & V
\end{array}\right], \quad \Delta_{4}=\left[\begin{array}{llll}
A & 0 & A_{d} & 0
\end{array}\right] \\
& \Xi_{5}=\operatorname{diag}\left\{-\bar{d}_{1}^{-1} Z_{1},-\bar{d}_{2}^{-1} Z_{2},-\bar{d}^{-1} Z_{2},-\bar{d}^{-1} M\right\} \text {, } \\
& \Delta_{1}=P A+A^{T} P+Q_{1}+R \text {, } \\
& \Delta_{2}=-\left(1-\tau_{1}\right)\left(Q_{1}-Q_{2}\right) \\
& \Delta_{3}=-\left(1-\tau_{1}-\tau_{2}\right) Q_{2}, \quad \bar{d}=\bar{d}_{1}+\bar{d}_{2} .
\end{aligned}
$$

Remark 1 Theorem 1 presents a new stability criterion for system $\Sigma$ with two successive time-varying delay components. This criterion is derived by defining the new Lyapunov-Krasovskii functional in (7), which makes full use of the information about $d_{1}(t)$ and $d_{2}(t)$. It is also worth mentioning that some novel techniques have been exploited in the calculation of the time derivative of $V(t)$, for example, no system transformation has been performed to the original system and thus there is no need to seek upper bounds of the inner product between two vectors, which has the potential to yield less conservative results. 
In the following, we further extend the above idea to an important case. More specifically, we assume the two successive delay components $d_{1}(t)$ and $d_{2}(t)$ have very different properties in that $d_{1}(t)$ and $d_{2}(t)$ are assumed to be constant and non-differentiable respectively. Thus the assumption in (4) reads

$$
d_{1}(t) \equiv \bar{d}_{1}<\infty, \quad 0 \leq d_{2}(t) \leq \bar{d}_{2}<\infty .
$$

As can be seen in the next section, this case is much related to the model we use for network based control.

Corollary 1 System $\Sigma$ in (6) with delays $d_{1}(t)$ and $d_{2}(t)$ satisfying (10) is asymptotically stable if there exist matrices $P>0, Q \geq 0, R \geq 0, Z_{i}>0, i=1,2, M>0$, and $S, T$, $U, V$ satisfying

$$
\left[\begin{array}{cc}
\bar{\Xi}_{1}+\Xi_{2}+\Xi_{2}^{T}+\Xi_{3} & \Xi_{4} \\
* & \Xi_{5}
\end{array}\right]<0,
$$

where $\bar{\Xi}_{1}=\Xi_{1}+\operatorname{diag}\left\{\left(Q-Q_{1}\right),\left(-\Delta_{2}-Q\right),-\Delta_{3}, 0\right\}$, and $\Xi_{i}, i=2, \ldots, 5, \Delta_{2}, \Delta_{3}$ are given in (9).

\section{2 $\mathscr{H}_{\infty}$ Performance Analysis}

In this subsection, we investigate the problem of $\mathscr{H}_{\infty}$ performance analysis for systems with two successive delay components in the state. Consider the following system:

$$
\begin{aligned}
\bar{\Sigma}: \quad \dot{x}(t) & =A x(t)+A_{d} x\left(t-d_{1}(t)-d_{2}(t)\right)+E w(t), \\
y(t) & =C x(t)+C_{d} x\left(t-d_{1}(t)-d_{2}(t)\right)+F w(t), \\
x(t) & =\phi(t), \quad t \in[-\bar{d}, 0] .
\end{aligned}
$$

Here $x(t), \phi(t), d_{1}(t)$ and $d_{2}(t)$ are the same as those in the above subsection; $w(t) \in \mathbb{R}^{l}$ is the disturbance input which belongs to $L_{2}[0, \infty) ; A, A_{d}, B, C, C_{d}, D$ are system matrices. Our objective is to investigate under what condition system $\bar{\Sigma}$ in (12) is asymptotically stable with an $\mathscr{H}_{\infty}$ disturbance attention level $\gamma$, that is, $\|y\|_{2}<\gamma\|w\|_{2}$ for all nonzero $w \in$ $L_{2}[0, \infty)$ under zero initial condition. We first consider the assumption in (4).

Theorem 2 System $\bar{\Sigma}$ in (12) with delays $d_{1}(t)$ and $d_{2}(t)$ satisfying (4) is asymptotically stable with an $\mathscr{H}_{\infty}$ disturbance attention level $\gamma$ if there exist matrices $P>0$, $Q_{1} \geq Q_{2} \geq 0, R \geq 0, Z_{1} \geq Z_{2}>0, M>0$, and $S, T, U$, $V$ satisfying

$$
\left[\begin{array}{cc}
\Psi_{1}+\Psi_{2}+\Psi_{2}^{T}+\Psi_{3}+\Psi_{4}+\Psi_{5} & \Xi_{4} \\
* & \Xi_{5}
\end{array}\right]<0,
$$

where $\Xi_{4}$ and $\Xi_{5}$ are given in (9),

$$
\begin{aligned}
& \Psi_{1}=\left[\begin{array}{ccccc}
\Delta_{1} & 0 & P A_{d} & 0 & P E \\
* & \Delta_{2} & 0 & 0 & 0 \\
* & * & \Delta_{3} & 0 & 0 \\
* & * & * & -R & 0 \\
* & * & * & * & 0
\end{array}\right], \\
& \Psi_{2}=\left[\begin{array}{lllll}
S+V & T-S & U-T & -U-V & 0
\end{array}\right], \\
& \Psi_{3}=\Delta_{5}^{T}\left[\bar{d}_{1} Z_{1}+\bar{d}_{2} Z_{2}+\left(\bar{d}_{1}+\bar{d}_{2}\right) M\right] \Delta_{5}, \\
& \Psi_{4}=\left[\begin{array}{lllll}
C & 0 & C_{d} & 0 & F
\end{array}\right]^{T}\left[\begin{array}{lllll}
C & 0 & C_{d} & 0 & F
\end{array}\right], \\
& \Psi_{5}=\operatorname{diag}\left\{0,0,0,0,-\gamma^{2} I\right\} \text {, } \\
& \Delta_{5}=\left[\begin{array}{lllll}
A & 0 & A_{d} & 0 & E
\end{array}\right] \text {, }
\end{aligned}
$$

and $\Delta_{i}, i=1,2,3$, are given in (9).

For the special case in (10), we have the following corollary.

Corollary 2 System $\bar{\Sigma}$ in (12) with delays $d_{1}(t)$ and $d_{2}(t)$ satisfying (10) is asymptotically stable with an $\mathscr{H}_{\infty}$ disturbance attention level $\gamma$ if there exist matrices $P>0, Q \geq 0$, $R \geq 0, Z_{i}>0, i=1,2, M>0$, and $S, T, U, V$ satisfying

$$
\left[\begin{array}{cc}
\bar{\Psi}_{1}+\Psi_{2}+\Psi_{2}^{T}+\Psi_{3}+\Psi_{4}+\Psi_{5} & \Xi_{4} \\
* & \Xi_{5}
\end{array}\right]<0
$$

where $\bar{\Psi}_{1}=\Psi_{1}+\operatorname{diag}\left\{\left(Q-Q_{1}\right),\left(-\Delta_{2}-Q\right),-\Delta_{3}, 0,0\right\}$, $\Xi_{4}, \Xi_{5}, \Delta_{2}, \Delta_{3}$ are given in (9) and $\Psi_{i}, i=2, \ldots, 5$, are given in (14).

\subsection{Illustrative Example}

We use a numerical example to illustrate the advantage of the proposed new model and the developed stability condition.

Example 1 Consider system $\Sigma$ in (6) with the following parameters (borrowed from [4, 15]):

$$
A=\left[\begin{array}{cc}
-0.2 & 0 \\
0 & -0.09
\end{array}\right], \quad A_{d}=\left[\begin{array}{cc}
-1 & 0 \\
-1 & -1
\end{array}\right]
$$

Suppose we know that $\dot{d}_{1}(t) \leq 0.1, \quad \dot{d}_{2}(t) \leq 0.8$. Our purpose is to find the upper bound $\bar{d}_{1}$ of delay $d_{1}(t)$, or $\bar{d}_{2}$ of $d_{2}(t)$, when the other is known, below which the system is asymptotically stable. By combining the two delay components together, some existing stability results can be applied to this system. The calculation results obtained by Theorem 1 in this paper, Theorem 2 in [19], Theorem 1 in [15], Theorem 3.2 in [17], Corollary 1 in [16] and Theorem 1 in [4] are listed in Table 1 for different cases. It can be seen from the table that Theorem 1 in this paper yields the least conservative stability test than the other single delay approaches, showing the advantage of our approach.

\section{Application to Network Based Control}

\subsection{Problem Formulation}

In this section, we apply the results obtained above to the problem of $\mathscr{H}_{\infty}$ control for networked control systems. Consider a typical networked control system shown in Figure 1. Suppose the physical plant is given by the following linear system:

$$
\begin{aligned}
& \dot{x}(t)=A x(t)+B u(t)+E w(t), \\
& y(t)=C x(t)+D u(t)+F w(t) .
\end{aligned}
$$

Here $x(t) \in \mathbb{R}^{n}$ is the state vector; $u(t) \in \mathbb{R}^{p}$ is the control input; $w(t) \in \mathbb{R}^{l}$ is the disturbance input which belongs to $L_{2}[0, \infty) ; y(t) \in \mathbb{R}^{q}$ is the output; and $A, B, C, D, E, F$ are system matrices with appropriate dimensions.

In Figure 1, it is assumed that the sampler is clock-driven, while the quantizer, controller and actuator are eventdriven. The sampling period is assumed to be $h$ where 


\begin{tabular}{c|c|c|c|c|c|c}
\hline & \multicolumn{3}{|c|}{ Delay bound $\bar{d}_{2}$ for given $\bar{d}_{1}$} & \multicolumn{3}{c}{ Delay bound $\bar{d}_{1}$ for given $\bar{d}_{2}$} \\
\hline & $\bar{d}_{1}=1$ & $\bar{d}_{1}=1.2$ & $\bar{d}_{1}=1.4$ & $\bar{d}_{2}=0.1$ & $\bar{d}_{2}=0.2$ & $\bar{d}_{2}=0.3$ \\
\hline By Theorem 1 & 0.512 & 0.406 & 0.320 & 2.300 & 1.779 & 1.453 \\
\hline By [19] & 0.364 & 0.164 & infeasible & 1.264 & 1.164 & 1.064 \\
\hline By [15] & 0.364 & 0.164 & infeasible & 1.264 & 1.164 & 1.064 \\
\hline By [4] & 0.364 & 0.164 & infeasible & 1.264 & 1.164 & 1.064 \\
\hline By [17] & infeasible & infeasible & infeasible & 0.315 & 0.215 & 0.115 \\
\hline By [16] & infeasible & infeasible & infeasible & 0.222 & 0.122 & 0.022 \\
\hline
\end{tabular}

Table 1 Calculated delay bounds for different cases

$h$ is a positive real constant. In addition, it is assumed that the state variable $x(t)$ is measurable, and the measurements of $x(t)$ are firstly quantized via a quantizer, and then transmitted with a single packet. The quantizer is denoted as $f(\cdot)=\left[\begin{array}{llll}f_{1}(\cdot) & f_{2}(\cdot) & \cdots & f_{n}(\cdot)\end{array}\right]^{T}$, which is assumed to be symmetric. In this paper, we are interested in the logarithmic static and time-invariant quantizer. For each $f_{j}(\cdot)$, the set of quantized levels is described by $\mathscr{U}_{j}=\left\{ \pm u_{i}^{(j)}, \quad i=0, \pm 1, \pm 2, \ldots\right\} \cup\{0\} . \quad$ According to $[3,5]$, for the logarithmic quantizer, the associated quantizer $f_{j}(\cdot)$ is defined as follows:

$f_{j}(v)= \begin{cases}u_{i}^{(j)}, & \text { if } \frac{1}{1+\sigma_{j}} u_{i}^{(j)}<v \leq \frac{1}{1-\sigma_{j}} u_{i}^{(j)}, \quad v>0 \\ 0, & \text { if } v=0, \\ -f_{j}(-v), & \text { if } v<0,\end{cases}$

where $\sigma_{j}=\frac{1-\rho_{j}}{1+\rho_{j}}$ with $\rho_{j}$ being the quantization density.

Now denote the updating instants of the zero-order hold $(\mathrm{ZOH})$ as $t_{k}, k=1, \ldots, \infty$, and suppose that the updating signal (successfully transmitted signal from the sampler to the controller and to the $\mathrm{ZOH}$ ) at the instant $t_{k}$ has experienced signal transmission delays $\eta_{k}\left(\eta_{k}=\tau_{k}+d_{k}\right.$ where $\tau_{k}$ is the delay from the quantizer to the controller and $d_{k}$ is the delay from the controller to the $\mathrm{ZOH}$ ). Therefore, considering the behavior of the $\mathrm{ZOH}$, the state-feedback controller takes the following form:

$$
u(t)=K f\left(x\left(t_{k}-\eta_{k}\right)\right), \quad t_{k} \leq t<t_{k+1},
$$

where $K$ is the state-feedback control gain, and $t_{k+1}$ is the next updating instant of the $\mathrm{ZOH}$ after $t_{k}$.

A natural assumption on the network induced delays $\eta_{k}$ can be made as $\eta_{m} \leq \eta_{k} \leq \eta_{M}$, where $\eta_{m}$ and $\eta_{M}$ denote the minimum and the maximum delays respectively. In addition, at the updating instant $t_{k}$ the number of accumulated data packet dropouts since the last updating instant $t_{k-1}$ is denoted as $\delta_{k}$. We assume $\delta_{k} \leq \bar{\delta}$ for all $k$. Then, we have

$$
h+\eta_{m} \leq t_{k+1}-t_{k} \leq \eta_{M}+(\bar{\delta}+1) h,
$$

which implies that the interval between any two successive updating instants is upper bounded by $\eta_{M}+(\bar{\delta}+1) h$ and lower bounded by $h+\eta_{m}$.

Remark 2 It is worth noting that the assumption on the network induced delays $\eta_{k}$ made above is more general than those in [20, 21]. The main difference lies in the lower bound we introduced. By assuming $\eta_{m}=0$, the assumption is the same as those in [20, 21]. The introduction of the lower bound $\eta_{m}$ will be shown later, via a numerical example, to be advantageous for reducing conservativeness by utilizing the idea of successive delay components developed in the above section.

Therefore, from (15)-(17) we obtain the following closedloop system:

$$
\begin{aligned}
\dot{x}(t)= & A x(t)+B K f\left(x\left(t_{k}-\eta_{k}\right)\right)+E w(t), \\
y(t)= & C x(t)+D K f\left(x\left(t_{k}-\eta_{k}\right)\right)+F w(t), \\
& t_{k} \leq t<t_{k+1} .
\end{aligned}
$$

Remark 3 It is important to note that in (17), $t_{k}$ refers to the updating instant of the ZOH. While in [20], the controller is expressed as $u(t)=K \bar{x}\left(t_{k}\right), \quad t_{k} \leq t<t_{k+1}$, with $t_{k}$ standing for the sampling instant. It should be noted that when the controller and actuator are event-driven, we cannot use the sampling instant to model the behavior of the $\mathrm{ZOH}$. The reason is that the signal transmission delays may not necessarily be integer multiples of the sampling period, and thus the $\mathrm{ZOH}$ may be updated between sampling instants. By using the updating instant in this paper, we do not need to synchronize the $\mathrm{ZOH}$ and the sampler, and thus the networked control model formulated here is essentially different from that in [20] and is more general, though they appear to be similar.

\section{2 $\mathscr{H}_{\infty}$ Controller Design}

It is noted that the closed-loop system in (19) is in the form of a sampled-data system. As the time sequence $\left\{t_{k}\right\}$ depends on both the network induced delays and data packet dropouts, the period $t_{k+1}-t_{k}$ for the sampled-data system in (19) is variable and uncertain. From (18) we know that $t_{k+1}-t_{k}$ is upper bounded by $\eta_{M}+(\bar{\delta}+1) h$ and lower bounded by $h+\eta_{m}$.

Now, let us represent $t_{k}-\eta_{k}$ in (19) as

$$
t_{k}-\eta_{k}=t-t+t_{k}-\eta_{m}+\eta_{m}-\eta_{k}=t-\eta_{m}-\eta(t),
$$

where $\eta(t)=t-t_{k}+\left(\eta_{k}-\eta_{m}\right)$. Then, from (18) we have

$$
0 \leq \eta(t) \leq \kappa
$$

where $\kappa=2 \eta_{M}-\eta_{m}+(\bar{\delta}+1) h$. By substituting (20) into (19) we obtain

$$
\begin{aligned}
& \dot{x}(t)=A x(t)+B K f\left(x\left(t-\eta_{m}-\eta(t)\right)\right)+E w(t), \\
& y(t)=C x(t)+D K f\left(x\left(t-\eta_{m}-\eta(t)\right)\right)+F w(t) .
\end{aligned}
$$


In addition, we denote $v=\eta_{m}+\kappa$.

Remark 4 In the above transformed system, $\eta_{m}$ is a constant delay, and $\eta(t)$ is a non-differentiable time-varying delay with bound $\kappa$. Our main idea in the above transformation is to represent the sampled-data system in (19) as a continuous time system with two successive delay components in the state, which takes a very similar form as system $\Sigma$ in (6) with the assumption in (10). The $\mathscr{H}_{\infty}$ control problem will be solved based on this new model.

Theorem 3 Consider the networked control system in Figure 1. Given a positive constant $\gamma$, there exists a statefeedback controller in the form of (17) such that the closedloop system in (19) is asymptotically stable with an $\mathscr{H}_{\infty}$ disturbance attention level $\gamma$ if there exist matrices $\bar{P}>0$, $\bar{Q} \geq 0, \bar{R} \geq 0, \bar{Z}_{i}>0, i=1,2, \bar{M}>0, \bar{K}, \bar{S}, \bar{T}, \bar{U}, \bar{V}$, and $a$ diagonal matrix $\bar{W}>0$ satisfying

$$
\left[\begin{array}{cccccc}
\Delta_{8} & \Pi_{3} & \Pi_{5} H & \Pi_{7} & \Pi_{8} & \Pi_{10} \\
* & \Pi_{4} & 0 & 0 & 0 & 0 \\
* & * & \Pi_{6} & 0 & \Pi_{9} & 0 \\
* & * & * & -I & D \bar{K} & 0 \\
* & * & * & * & \bar{W}-2 \bar{P} & 0 \\
* & * & * & * & * & -\Lambda^{-2} \bar{W}
\end{array}\right]<0,
$$

where

$$
\begin{aligned}
& \Pi_{1}=\left[\begin{array}{ccccc}
A \bar{P}+\bar{P} A^{T}+\bar{Q}+\bar{R} & 0 & B \bar{K} & 0 & E \\
* & -\bar{Q} & 0 & 0 & 0 \\
* & * & 0 & 0 & 0 \\
* & * & * & -\bar{R} & 0 \\
* & * & * & * & 0
\end{array}\right], \\
& \Pi_{2}=\left[\begin{array}{llllll}
\bar{S}+\bar{V} & \bar{T}-\bar{S} & \bar{U}-\bar{T} & -\bar{U}-\bar{V} & 0
\end{array}\right], \\
& \Pi_{3}=\left[\begin{array}{llll}
\bar{S} & \bar{T} & \bar{U} & \bar{V}
\end{array}\right], \quad H=\left[\begin{array}{lll}
I & I & I
\end{array}\right], \\
& \Pi_{6}=\operatorname{diag}\left\{-\eta_{m}^{-1} \bar{Z}_{1},-\kappa^{-1} \bar{Z}_{2},-v^{-1} \bar{M}\right\}, \\
& \Pi_{9}=H^{T} B \bar{K}, \quad \Delta_{8}=\Pi_{1}+\Pi_{2}+\Pi_{2}^{T}+\Psi_{5}, \\
& \Pi_{4}=\operatorname{diag}\left\{\eta_{m}^{-1}\left(\bar{Z}_{1}-2 \bar{P}\right), \kappa^{-1}\left(\bar{Z}_{2}-2 \bar{P}\right),\right. \\
& \left.v^{-1}\left(\bar{Z}_{2}-2 \bar{P}\right), v^{-1}(\bar{M}-2 \bar{P})\right\} \text {, } \\
& \Pi_{5}=\left[\begin{array}{c}
\bar{P} A^{T} \\
0 \\
\bar{K}^{T} B^{T} \\
0 \\
E^{T}
\end{array}\right], \Pi_{7}=\left[\begin{array}{c}
\bar{P} C^{T} \\
0 \\
\bar{K}^{T} D^{T} \\
0 \\
F^{T}
\end{array}\right], \Pi_{8}=\left[\begin{array}{c}
B \bar{K} \\
0 \\
0 \\
0 \\
0
\end{array}\right], \\
& \Pi_{10}=\left[\begin{array}{lllll}
0 & 0 & \bar{P} & 0 & 0
\end{array}\right]^{T},
\end{aligned}
$$

and $\Psi_{5}$ is given in (14). Moreover, if the above condition is feasible, a desired controller gain matrix in the form of (17) is given by $K=\bar{K} \bar{P}^{-1}$.

\subsection{Illustrative Example}

Example 2 Suppose the physical plant in Figure 1 is the satellite system, considered in [2]. The satellite system consists of two rigid bodies joined by a flexible link. This link is modelled as a spring with torque constant $k$ and viscous damping $f$. Denoting the yaw angles for the two bodies (the main body and the instrumentation module) by $\theta_{1}$ and $\theta_{2}$, the control torque by $u(t)$, the moments of inertia of the two bodies by $J_{1}$ and $J_{2}$. Assume the output is the angular position $\theta_{2}(t)$. Here we choose $J_{1}=J_{2}=1, k=0.09$ and $f=0.04$. Then, the corresponding matrices described in Section 3 are given by

$$
\begin{aligned}
A & =\left[\begin{array}{cccc}
0 & 0 & 1 & 0 \\
0 & 0 & 0 & 1 \\
-0.3 & 0.3 & -0.004 & 0.004 \\
0.3 & -0.3 & 0.004 & -0.004
\end{array}\right], \\
B & =\left[\begin{array}{llll}
0 & 0 & 1 & 0
\end{array}\right]^{T}, \quad E=\left[\begin{array}{llll}
0 & 0 & 0 & 0.1
\end{array}\right]^{T}, \\
C & =\left[\begin{array}{llll}
0 & 1 & 0 & 0
\end{array}\right], \quad D=0, \quad F=0 .
\end{aligned}
$$

It is assumed that: the sampling period $h=10 \mathrm{~ms}$; the network induced delay bounds are given by $\eta_{m}=10 \mathrm{~ms}$ and $\eta_{M}=20 \mathrm{~ms}$; the maximum number of data packet dropouts $\bar{\delta}=2$. In addition, the parameters for the quantizer $f(\cdot)$ are assumed to be $\rho_{1}=\rho_{2}=\rho_{3}=\rho_{4}=0.9$.

The eigenvalues of $A$ are $-0.04+0.4224 j,-0.0400-$ $0.4224 \mathrm{j}, 0$, 0; thus the above system is not stable. By using Theorem 3 (minimizing $\gamma$ in (22)), the gain matrix for the state-feedback controller in (17) is given by $K=$ $\left[\begin{array}{llll}-1.1789 & -1.3096 & -1.6629 & -7.3974\end{array}\right]$, and the obtained minimum guaranteed $\mathscr{H}_{\infty}$ performance is $\gamma^{*}=$ 0.7864 .

We first illustrate that the closed-loop system is asymptotically stable under the above obtained controller. The state responses are depicted in Figure 2, from which we can see that all four state components converge to zero. In the simulation, the network induced delays and the data packet dropouts are generated randomly (evenly distributed within their ranges) according to the above assumption. The computed control inputs arriving at the $\mathrm{ZOH}$ are shown in Figure 3, where we can see the discontinuous holding behavior of the control inputs.

Next, we illustrate the $\mathscr{H}_{\infty}$ performance of the closed-loop system. To this end, let us assume zero initial conditions, and select a set of input signals as follows:

$$
w(t)= \begin{cases}\sin 0.2 t, & 5 \leq t \leq 15 s, \\ 0, & \text { otherwise. }\end{cases}
$$

In the simulation, by calculation, $\|w\|_{2}=2.5468,\|y\|_{2}=$ 1.3537, which yields $\frac{\|y\|_{2}}{\|w\|_{2}}=0.5315<\gamma^{*}=0.7864$, showing the effectiveness of the $\mathscr{H}_{\infty}$ controller design.

\section{Conclusions}

This paper has proposed a new model on time-delay systems, which contains multiple successive delay components in the state. New results on stability and $\mathscr{H}_{\infty}$ performance are proposed by exploiting a new LyapunovKrasovskii functional and by making use of novel techniques for time-delay systems. Moreover, the proposed new model has been utilized to model networked control systems with simultaneous consideration of network induced delays, data packet dropouts and measurement quantization. Illustrative examples have been presented to show the advantage and applicability of the proposed new timedelay model and network based controller design method. 


\section{REFERENCES}

[1] P. Antsaklis and J. Baillieul. Guest editorial. Special issue on networked control systems. IEEE Trans. Automat. Control, 49(9):1421-1423, 2004.

[2] R. M. Biernacki, H. Hwang, and S. P. Battacharyya. Robust stability with structured real parameter perturbations. IEEE Trans. Automat. Control, 32:495-506, 1987.

[3] N. Elia and S. K. Mitter. Stabilization of linear systems with limited information. IEEE Trans. Automat. Control, 46(9):1384-1400, 2001.

[4] E. Fridman and U. Shaked. Delay-dependent stability and $H_{\infty}$ control: constant and time-varying delays. Int. J. Control, 76(1):48-60, 2003.

[5] M. Fu and L. Xie. The sector bound approach to quantized feedback control. IEEE Trans. Automat. Control, 50(11):1698-1711, 2005.

[6] H. Gao, J. Lam, and C. Wang. Robust energy-to-peak filter design for stochastic time-delay systems. Systems \& Control Letters, 55(2):101-111, 2006.

[7] H. Gao, J. Lam, C. Wang, and Q. Wang. Hankel norm approximation of linear systems with time-varying delay: Continuous and discrete cases. Int. J. Control, 77:15031520, 2004.

[8] H. Gao, J. Lam, C. Wang, and Y. Wang. Delay-dependent output-feedback stabilisation of discrete-time systems with time-varying state delay. IEE Proc. Part D: Control Theory Appl., 151:691-698, 2004.

[9] H. Gao and C. Wang. Comments and further results on 'A descriptor system approach to $H_{\infty}$ control of linear timedelay systems'. IEEE Trans. Automat. Control, 48(3):520$525,2003$.

[10] H. Gao and C. Wang. Delay-dependent robust $H_{\infty}$ and $L_{2}-$ $L_{\infty}$ filtering for a class of uncertain nonlinear time-delay systems. IEEE Trans. Automat. Control, 48(9):1661-1666, 2003.

[11] H. Gao and C. Wang. Robust $L_{2}-L_{\infty}$ filtering for uncertain systems with multiple time-varying state delays. IEEE Trans. Circuits and Systems (I), 50(4):594-599, 2003.

[12] H. Gao and C. Wang. A delay-dependent approach to robust $H_{\infty}$ filtering for uncertain discrete-time state-delayed systems. IEEE Trans. Signal Processing, 52(6):1631-1640, 2004.

[13] G. C. Goodwin, H. Haimovich, D. E. Quevedo, and J. S. Welsh. A moving horizon approach to networked control system design. IEEE Trans. Automat. Control, 49(9):14271445, 2004.

[14] K. Gu, V. L. Kharitonov, and J. Chen. Stability of TimeDelay Systems. Springer-Verlag, Berlin, Germany, 2003.

[15] X. J. Jing, D. L. Tan, and Y. C. Wang. An LMI approach to stability of systems with severe time-delay. IEEE Trans. Automat. Control, 49(7):1192-1195, 2004.

[16] J.-H. Kim. Delay and its time-derivative dependent robust stability of time-delayed linear systems with uncertainty. IEEE Trans. Automat. Control, 46(5):789-792, 2001.

[17] Y. S. Lee, Y. S. Moon, W. H. Kwon, and K. H. Lee. Delaydependent robust $H_{\infty}$ control for uncertain systems with time-varying state-delay. In Proc. 40th Conf. Decision Control, pages 3208-3213, Orlando, FL, 2001.

[18] J. P. Richard. Time-delay systems: an overview of some recent advances and open problems. Automatica, 39(10):1667-1694, 2003.

[19] M. Wu, Y. He, J. H. She, and G. P. Liu. Delay-dependent criteria for robust stability of time-varying delay systems. Automatica, 40:1435-1439, 2004.

[20] M. Yu, L. Wang, and T. Chu. Sampled-data stabilization of networked control systems with nonlinearity. IEE Proc. Part D: Control Theory Appl., 152(6):609-614, 2005.

[21] M. Yu, L. Wang, T. Chu, and F. Hao. Stabilization of networked control systems with data packet dropout and transmission delays: continuous-time case. European J. Contr. 11(1):40-55, 2005.

[22] W. Zhang, M. Branicky, and S. Phillips. Stability of networked control systems. IEEE Control Systems Magzine, 21:84-99, 2001

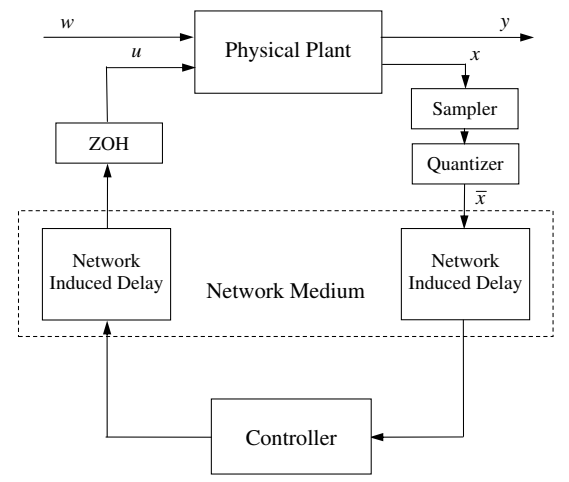

Figure 1: A typical networked control system

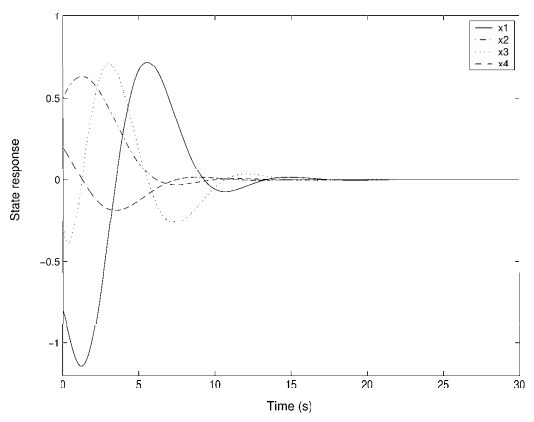

Figure 2: State response

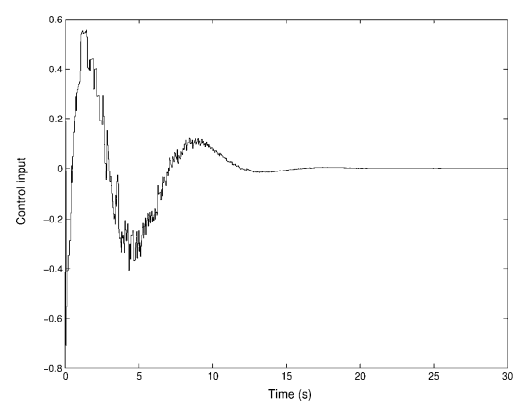

Figure 3: Control input signal 